\title{
On a class of analytic functions associated to a complex domain concerning q-differential-difference operator
}

\author{
Rabha W. Ibrahim ${ }^{1,2^{*}}$ and Maslina Darus ${ }^{3}$
}

\section{"Correspondence:}

rabhaibrahim@yahoo.com;

rabhaibrahim@tdtu.edu.vn

'Informetrics Research Group, Ton

Duc Thang University, Ho Chi Minh City, Vietnam

${ }^{2}$ Faculty of Mathematics \& Statistics, Ton Duc Thang University, Ho Chi

Minh City, Vietnam

Full list of author information is available at the end of the article

\begin{abstract}
In our current investigation, we apply the idea of quantum calculus and the convolution product to amend a generalized Salagean q-differential operator. By considering the new operator and the typical version of the Janowski function, we designate definite new classes of analytic functions in the open unit disk. Significant properties of these modules are considered, and recurrent sharp consequences and geometric illustrations are realized. Applications are considered to find the existence of solutions of a new class of q-Briot-Bouquet differential equations.
\end{abstract}

MSC: $30 C 45 ; 30 C 50$

Keywords: Univalent function; Differential operator; Unit disk; Analytic function; Fractional calculus; q-calculus; Subordination and superordination

\section{Introduction}

The q-calculus motivates to build a new method of q-special functions, new differential and difference operators and generalized well-known differential and difference equations. The structure of q-calculus improves different modules of orthogonal polynomials and functions as regards the procedure of their traditional complements. The joining between equilibriums of differential formulas (equations, operators and inequalities) and their solutions is one of the most beneficial and well-designed tools for studying properties of the special functions in mathematical analysis and mathematical physics. The consequence of these concerns in applications to solve physical problems need not be strained. The q-operators usually realize q-difference equations (which may include derivatives). We show the close connection between these operators of q-difference equations. In certain studies, we shall present a technique for developing and understanding from a geometric viewpoint numerous properties and characteristics of q-operators.

The theory of q-calculus mainly was recently developed. Studies of q-difference equations were widely performed essentially by Carmichael [1], Jackson [2], Mason [3], and Trjitzinsky [4]. Investigation concerning the geometric function theory and q-theory together was first given by Ismail et al.[5]. Many differential and integral operators can be recorded in terms of convolution, such as the Sàlàgean differential operator [6], Al-Oboudi

(c) The Author(s) 2019. This article is licensed under a Creative Commons Attribution 4.0 International License, which permits use sharing, adaptation, distribution and reproduction in any medium or format, as long as you give appropriate credit to the original author(s) and the source, provide a link to the Creative Commons licence, and indicate if changes were made. The images or other third party material in this article are included in the article's Creative Commons licence, unless indicated otherwise in a credit line to the material. If material is not included in the article's Creative Commons licence and your intended use is not permitted by statutory regulation or exceeds the permitted use, you will need to obtain permission directly from the copyright holder. To view a copy of this licence, visit http://creativecommons.org/licenses/by/4.0/. 
differential operator [7], and the generalized differential operator [8]. It is an advantage that the method of convolution aids investigators in extra investigation of the geometric properties for some well-known classes of analytic univalent functions.

Newly, Naeem et al. [9] announced investigations of classes linking the Sàlàgean qdifferential operator. By joining the q-calculus and the generalized Sàlàgean differential operator [8], we present a new generalized q-operator called the generalized Sàlàgean qdifferential operator. By using the new formula of this operator, we formulate some new classes and investigate the geometric consequences of them.

\section{Precursory}

We intend to require and assume the following throughout this study. A function $v \in \Lambda$ is known as univalent in $\mathbb{U}$ (the open unit disk) if it definitely obeys: if $\xi_{1} \neq \xi_{2}$ in $\mathbb{U}=\{z \in$ $\mathbb{C}:|z|<1\}$ then $\psi\left(\xi_{1}\right) \neq \psi\left(\xi_{2}\right)$ or equivalently, if $v\left(\xi_{1}\right)=v\left(\xi_{2}\right)$ then $\xi_{1}=\xi_{2}$. Without loss of generality, we suggest the letter $\Lambda$ for our univalent functions as regards the construction

$$
v(z)=z+\sum_{n=2}^{\infty} \vartheta_{n} z^{n}, \quad z \in \mathbb{U} .
$$

We let $\mathcal{S}$ denote the class of such functions $v \in \Lambda$ as are univalent in $\mathbb{U}$.

A function $v \in \mathcal{S}$ is recalled starlike w.r.t. $(0,0)$ in $\mathbb{U}$ if the linear cut associating the origin to every other point of $v(z),|z|=r<1$ is set entirely in $v(z),|z|=r<1$ (every point of $v(z)$ be observable from the origin). A function $v \in \mathcal{S}$ is called convex in $\mathbb{U}$ if the linear slice fitting together any two points of $v(z),|z|=r<1$ is set entirely in $v(z),|z|=r<1$ or a function $v \in \mathcal{S}$ is convex in $\mathbb{U}$ if it is starlike. We address the class of functions $v \in \mathcal{S}$ that are starlike with respect to the origin by $\mathcal{S}^{*}$ and convex in $\mathbb{U}$ by $\mathcal{C}$.

Connected to the classes $\mathcal{S}^{*}$ and $\mathcal{C}$, we address the class $\mathcal{P}$ of all analytic functions $v$ in $\mathbb{U}$ with a positive real part in $\mathbb{U}$ and $v(0)=1$. In fact $\psi \in \mathcal{S}^{*}$ if and only if $z v^{\prime}(z) / v(z) \in \mathcal{P}$ and $v \in \mathcal{C}$ if and only if $1+z v^{\prime \prime}(z) / v^{\prime}(z) \in \mathcal{P}$. Extensively, for a positive number $\sigma \in[0,1)$, we address the class $\mathcal{P}(\sigma)$ of analytic functions $v$ in $\mathbb{U}$ with $v(0)=1$ such that $\Re(v(z))>\sigma$ for all $z \in \mathbb{U}$. Note that [10] $\mathcal{P}\left(\sigma_{2}\right) \subset \mathcal{P}\left(\sigma_{1}\right) \subset \mathcal{P}(0) \equiv \mathcal{P}$ for $0<\sigma_{1}<\sigma_{2}$. According to [11], for two functions $v$ and $v \in \Lambda$, the function $v$ is subordinate to $v$, denoted by $v \prec v$, if there occurs a Schwarz function $\varsigma$ with $\varsigma(0)=0$ and $|\varsigma(z)|<1$ such that $v(z)=v(\varsigma(z))$ for all $z \in \mathbb{U}$. Clearly, $v(z) \prec v(z)$ is analogous to $v(0)=v(0)$ and $v(\mathbb{U}) \subset v(\mathbb{U})$.

It is advantageous stating that the method of convolution helps investigators in extra investigations of the geometric properties of analytic functions. For any non-negative integer $n$, the q-integer number $n$, symbolized by $[n, q]$, is formulated as $[n, q]=\frac{1-q^{n}}{1-q}$, where $[0, q]=0,[1, q]=1$ and $\lim _{q \rightarrow 1^{-}}[n, q]=n$. The q-difference operator of the analytic function $v$ is formulated by the construction

$$
\Delta_{q} v(z)=\frac{v(q z)-v(z)}{q z-z}, \quad z \in \mathbb{U}
$$

Clearly, $\Delta_{q} z^{n}=[n, q] z^{n-1}$ and for $v \in \Lambda$, we have

$$
\Delta_{q} v(z)=\sum_{n=1}^{\infty} \vartheta_{n}[n, q] z^{n-1}, \quad z \in \mathbb{U}, \vartheta_{1}=1
$$


For $v \in \Lambda$, Govindaraj and Sivasubramanian presented the Sàlàgean q-differential operator [12]

$$
S_{q}^{0} v(z)=v(z), \quad S_{q}^{1} v(z)=z \Delta_{q} v(z), \ldots, S_{q}^{k} v(z)=z \Delta_{q}\left(S_{q}^{k-1} v(z)\right)
$$

where $k$ is a positive integer. A calculation dependent on the formula of $\Delta_{q}$ shows $S_{q}^{k} v(z)=$ $v(z) * \Omega_{q}^{k}(z)$, where $*$ is the convolution product, $\Omega_{q}^{k}(z)=z+\sum_{n=2}^{\infty}[n, q]^{k} z^{n}$ and $S_{q}^{k} v(z)=$ $z+\sum_{n=2}^{\infty}[n, q]^{k} \vartheta_{n} z^{n}$. It is clear that

$$
\lim _{q \rightarrow 1^{-}} S_{q}^{k} v(z)=z+\sum_{n=2}^{\infty} n^{k} \vartheta_{n} z^{n}
$$

the normalized Sàlàgean differential operator [6].

For a function $v(z)$ and a constant $\kappa \in \mathbb{R}$, we construct the generalized q-Sàlàgean differential-difference operator (q-SDD) employing the idea of $\Delta_{q}$ as follows:

$$
\begin{aligned}
\mathcal{S}_{q}^{\kappa, 0} v(z) & =v(z) \\
\mathcal{S}_{q}^{\kappa, 1} v(z) & =z \Delta_{q} v(z)+\frac{\kappa}{2}(v(z)-v(-z)-2 z) \\
& =z+\sum_{n=2}^{\infty}\left([n, q]+\frac{\kappa}{2}\left(1+(-1)^{n+1}\right)\right) \vartheta_{n} z^{n}, \\
\mathcal{S}_{q}^{\kappa, 2} v(z) & =\mathcal{S}_{q}^{\kappa, 1}\left[\mathcal{S}_{q}^{\kappa, 1} v(z)\right]=z+\sum_{n=2}^{\infty}\left([n, q]+\frac{\kappa}{2}\left(1+(-1)^{n+1}\right)\right)^{2} \vartheta_{n} z^{\prime} n \\
\vdots & \mathcal{S}_{q}^{\kappa, k} v(z)=\mathcal{S}_{q}^{\kappa, 1}\left[\mathcal{S}_{q}^{\kappa, k-1} v(z)\right]=z+\sum_{n=2}^{\infty}\left([n, q]+\frac{\kappa}{2}\left(1+(-1)^{n+1}\right)\right)^{k} \vartheta_{n} z^{n} .
\end{aligned}
$$

Obviously, $\lim _{q \rightarrow 1^{-}} \mathcal{S}_{q}^{\kappa, k} v(z)$ implies the generalized Sàlàgean differential-difference operator [8], which is a special type of Dunkl operator with Dunkl constant $\kappa$ in the open unit disk [13]. Moreover, when $\kappa=0\left(\lim _{q \rightarrow 1^{-}} \mathcal{S}_{q}^{0, k} v(z)\right)$, we have the normalized Sàlàgean differential operator [6]. Finally, when $\kappa=0$, we have the q-Sàlàgean differential operator $\left(\mathcal{S}_{q}^{0, k} v(z)\right)$ (see [12]).

Based on the operator (2), we introduce the following classes.

Definition 2.1 A function $v \in \Lambda$ is in the class $S_{q}^{*}(\kappa, k, h)$ if and only if

$$
S_{q}^{*}(\kappa, k, h)=\left\{\psi \in \Lambda: \frac{z\left(\mathcal{S}_{q}^{\kappa, k} v(z)\right)^{\prime}}{\mathcal{S}_{q}^{\kappa, k} v(z)} \prec h(z), h \in \mathcal{C}\right\}
$$

- $S_{q}^{*}(\kappa, 0, h)=\mathcal{S}^{*}(h)$

- $S_{q}^{*}(\kappa, 0, h)=\mathcal{S}^{*}(h), h(z)=\frac{1+A z}{1+B z}($ see $[14-16])$;

- $S_{q}^{*}(\kappa, 0, h)=\mathcal{S}^{*}(h), h(z)=\frac{2}{1+e^{-z}}($ see $[17])$;

- $S_{q}^{*}(\kappa, 0, h)=\mathcal{S}^{*}(h), h(z)=\frac{1+\epsilon^{2} z^{2}}{1-\epsilon^{2}-\epsilon^{2} z^{2}}, \epsilon=\frac{1-\sqrt{5}}{2}$ (see $\left.[18,19]\right)$;

- $S_{q}^{*}(\kappa, 0, h)=\mathcal{S}^{*}(h), h(z)=1+\frac{\beta-\alpha}{\pi} i \log \left(\frac{1-\exp \left(2 \pi i\left(\frac{1-\alpha}{\beta-\alpha}\right)\right) z}{1-z}\right)$ (see [20]); 
- $S_{q}^{*}(\kappa, 0, h)=\mathcal{S}^{*}(h), h(z)=1+\frac{2}{\pi(1-\alpha)} i \log \left(\frac{1-\exp \left(\pi i(1-\alpha)^{2} z\right.}{1-z}\right)$ (see [21]);

- $S_{q}^{*}(\kappa, 0, h)=\mathcal{S}^{*}(h), h(z)=\sqrt{1+z}($ see $[22])$;

- $S_{q}^{*}(\kappa, 0, h)=\mathcal{S}^{*}(h), h(z)=1+\sin (z)$ (see [23]);

- $S_{q}^{*}(\kappa, 0, h)=\mathcal{S}^{*}(h), h(z)=1+\cos (z)$ (see [24]);

- $S_{q}^{*}(\kappa, 0, h)=\mathcal{S}^{*}(h), h(z)=\left(\frac{1+z}{1+\left(\frac{1-c}{c}\right) z}\right)^{\frac{1}{\mu}}, \mu \geq 1, c \geq 0.5$ (see [25]).

Definition 2.2 If $v \in \Lambda$, then $v \in \mathbb{J}_{q}^{\kappa, b}(A, B, k)$ if and only if

$$
\begin{aligned}
1+ & \frac{1}{b}\left(\frac{2 \mathcal{S}_{q}^{\kappa, k+1} v(z)}{\mathcal{S}_{q}^{\kappa, k} v(z)-\mathcal{S}_{q}^{\kappa, k} v(-z)}\right) \prec \frac{1+A z}{1+B z} \\
& (z \in \mathbb{U},-1 \leq B<A \leq 1, k=1,2, \ldots, b \in \mathbb{C} \backslash\{0\}, \kappa \in \mathbb{R}) .
\end{aligned}
$$

- $\kappa=0, q \rightarrow 1^{-} \Longrightarrow[26]$

- $\kappa=0, B=0, q \rightarrow 1^{-} \Longrightarrow[27]$

- $\kappa=0, A=1, B=-1, b=2, q \rightarrow 1^{-} \Longrightarrow[28]$;

- $q \rightarrow 1^{-} \Longrightarrow[8]$.

We shall study the geometric significance of the special classes $S_{q}^{*}(\lambda, k, h)$ and $\mathbb{J}_{q}^{\lambda, b}(A, B, k)$ by using the following preliminaries, which can be found in [11].

Lemma 2.1 Suppose the following data: $a \in \mathbb{C}$, a positive integer $n$ and

$$
\mathfrak{H}[\vartheta, n]=\left\{v: v(z)=\vartheta+\vartheta_{n} z^{n}+\vartheta_{n+1} z^{n+1}+\cdots\right\} .
$$

i. If $\wp \in \mathbb{R}$ then $\Re\left(v(z)+\wp z v^{\prime}(z)\right)>0 \Longrightarrow \Re(v(z))>0$. Moreover, if $\wp>0$ and $v \in \mathfrak{H}[1, n]$, then there are constants $\ell>0$ and $b>0$ with $b=b(\wp, \ell, n)$ so that

$$
v(z)+\wp z v^{\prime}(z) \prec\left[\frac{1+z}{1-z}\right]^{b} \Rightarrow v(z) \prec\left[\frac{1+z}{1-z}\right]^{\ell}
$$

ii. If $v \in[0,1)$ and $\psi \in \mathfrak{H}[1, n]$ then there is a constant $\ell>0$ with $\ell$ so that

$$
\Re\left(v^{2}(z)+2 v(z) \cdot z v^{\prime}(z)\right)>v \quad \Rightarrow \quad \Re(v(z))>\ell .
$$

iii. If $v \in \mathfrak{H}[\vartheta, n]$ with $\Re(\vartheta)>0$ then

$$
\mathfrak{R}\left(v(z)+z v^{\prime}(z)+z^{2} v^{\prime \prime}(z)\right)>0
$$

or for $\alpha: \mathbb{U} \rightarrow \mathbb{R}$ with

$$
\Re\left(v(z)+\alpha(z) \frac{z v^{\prime}(z)}{v(z)}\right)>0
$$

then $\Re(v(z))>0$. 


\section{Outcomes}

In this section, we study the geometric properties of the classes $S_{q}^{*}(\kappa, k, h)$ and $\mathbb{J}_{q}^{\kappa, b}(A, B, k)$ and the consequences of these classes for recent investigations by researchers.

Theorem 3.1 For $v \in \Lambda$ if one of the following statements is given:

- $\mathcal{S}_{q}^{\kappa, k} v(z)$ is of bounded boundary rotation;

- $v$ satisfies the subordination structure

$$
\left(\mathcal{S}_{q}^{\kappa, k} v(z)\right)^{\prime} \prec\left(\frac{1+z}{1-z}\right)^{b}, \quad b>0, z \in \mathbb{U} ;
$$

- $v$ fulfills the layout

$$
\Re\left(\left(\mathcal{S}_{q}^{\kappa, k} v(z)\right)^{\prime} \frac{\mathcal{S}_{q}^{\kappa, k} v(z)}{z}\right)>\frac{\varsigma}{2}, \quad \varsigma \in[0,1), z \in \mathbb{U},
$$

- $v$ obeys the relation

$$
\Re\left(z\left(\mathcal{S}_{q}^{\kappa, k} v(z)\right)^{\prime \prime}-\left(\mathcal{S}_{q}^{\kappa, k} v(z)\right)^{\prime}+2 \frac{\mathcal{S}_{q}^{\kappa, k} v(z)}{z}\right)>0,
$$

- $v$ admits the relation

$$
\Re\left(\frac{z\left(\mathcal{S}_{q}^{\kappa, k} v(z)\right)^{\prime}}{\mathcal{S}_{q}^{\kappa, k} v(z)}+2 \frac{\mathcal{S}_{q}^{\kappa, k} v(z)}{z}\right)>1,
$$

then $\frac{\mathcal{S}_{q}^{\kappa, k} v(z)}{z} \in \mathcal{P}(\sigma)$ for some $\sigma \in[0,1)$.

Proof Consider a function $\rho$ as follows:

$$
\rho(z)=\frac{\mathcal{S}_{q}^{\kappa, k} v(z)}{z} \Rightarrow z \rho^{\prime}(z)+\rho(z)=\left(\mathcal{S}_{q}^{\kappa, k} v(z)\right)^{\prime}
$$

By the first conclusion, $\mathcal{S}_{q}^{\kappa, k} v(z)$ is of bounded boundary rotation, it implies that $\Re\left(z \rho^{\prime}(z)+\right.$ $\rho(z))>0$. Thus, by Lemma 2.1.i, we obtain $\Re(\rho(z))>0$ which implies the first part of the theorem.

According to the second part, we have the subject subordination layout

$$
\left(\mathcal{S}_{q}^{\kappa, k} v(z)\right)^{\prime}=z \rho^{\prime}(z)+\rho(z) \prec\left[\frac{1+z}{1-z}\right]^{b}
$$

Now, according to Lemma 2.1.i, there is a constant $\ell>0$ with $b=b(\ell)$ accepting the subordination

$$
\frac{\mathcal{S}_{q}^{\kappa, k} v(z)}{z} \prec\left(\frac{1+z}{1-z}\right)^{\ell}
$$

This implies that

$$
\Re\left(\frac{\mathcal{S}_{q}^{\kappa, k} v(z)}{z}\right)>\sigma, \quad \sigma \in[0,1) .
$$


Continuing, we address the third part, which implies that

$$
\Re\left(\rho^{2}(z)+2 \rho(z) \cdot z \rho^{\prime}(z)\right)=2 \Re\left(\left(\mathcal{S}_{q}^{\kappa, k} v(z)\right)^{\prime} \frac{\mathcal{S}_{q}^{\kappa, k} v(z)}{z}\right)>\varsigma
$$

In virtue of Lemma 2.1.ii, there is a constant $\ell>0$ such that $\Re(\rho(z))>\ell$ and

$$
\rho(z)=\frac{\mathcal{S}_{q}^{\kappa, k} v(z)}{z} \in \mathcal{P}(\sigma), \quad \sigma \in[0,1)
$$

It follows from (4) that $\left.\Re\left(\mathcal{S}_{q}^{\kappa, k} v(z)\right)^{\prime}\right)>0$ and thus by the Noshiro-Warschawski and Kaplan theorems that $\mathcal{S}_{q}^{\kappa, k} v(z)$ is univalent and of bounded boundary rotation in $\mathbb{U}$.

By differentiating (3) and taking the real part, we have

$$
\Re\left(\rho(z)+z \rho^{\prime}(z)+z^{2} \rho^{\prime \prime}(z)\right)=\Re\left(z\left(\mathcal{S}_{q}^{\kappa, k} v(z)\right)^{\prime \prime}-\left(\mathcal{S}_{q}^{\kappa, k} v(z)\right)^{\prime}+2 \frac{\mathcal{S}_{q}^{\kappa, k} v(z)}{z}\right)>0
$$

Thus, in view of Lemma 2.1-ii, we attain $\Re\left(\frac{\mathcal{S}_{q}^{\kappa, k} v(z)}{z}\right)>0$.

By logarithmic differentiation (3) and taking the real part, we obtain the following:

$$
\Re\left(\rho(z)+\frac{z \rho^{\prime}(z)}{\rho(z)}+z^{2} \rho^{\prime \prime}(z)\right)=\Re\left(\frac{z\left(\mathcal{S}_{q}^{\kappa, k} v(z)\right)^{\prime}}{\mathcal{S}_{q}^{\kappa, k} v(z)}+2 \frac{\mathcal{S}_{q}^{\kappa, k} v(z)}{z}-1\right)>0
$$

Thus, according to Lemma 2.1-iii, where $\alpha(z)=1$, we get $\mathfrak{R}\left(\frac{\mathcal{S}_{q}^{\kappa, k} v(z)}{z}\right)>0$.

Theorem 3.2 Consider $v \in S_{q}^{*}(\kappa, k, h)$, where $h(z)$ is convex univalent function in $\mathbb{U}$. Then

$$
\mathcal{S}_{q}^{\kappa, k} v(z) \prec z \exp \left(\int_{0}^{z} \frac{h(ð(w))-1}{w} d w\right)
$$

where $\partial(z)$ is analytic in $\mathbb{U}$, with $\partial(0)=0$ and $|\partial(z)|<1$. Moreover, for $|z|=\chi, \mathcal{S}_{q}^{\kappa, k} v(z)$ fulfills the formula

$$
\exp \left(\int_{0}^{1} \frac{h ð(-\chi))-1}{\chi}\right) d \chi \leq\left|\frac{\mathcal{S}_{q}^{\kappa, k} v(z)}{z}\right| \leq \exp \left(\int_{0}^{1} \frac{h(ð(\chi))-1}{\chi}\right) d \chi
$$

Proof Since $\psi \in S_{q}^{*}(\kappa, k, h)$, we get

$$
\left(\frac{z\left(\mathcal{S}_{q}^{\kappa, k} v(z)\right)^{\prime}}{\mathcal{S}_{q}^{\kappa, k} v(z)}\right) \prec h(z), \quad z \in \mathbb{U}
$$

which leads to a Schwarz function with $\precsim(0)=0$ and $|ð(z)|<1$ satisfying the following equality:

$$
\left(\frac{z\left(\mathcal{S}_{q}^{\kappa, k} v(z)\right)^{\prime}}{\mathcal{S}_{q}^{\kappa, k} v(z)}\right)=h(ð(z)), \quad z \in \mathbb{U} .
$$


A calculation gives

$$
\left(\frac{\left(\mathcal{S}_{q}^{\kappa, k} \psi(z)\right)^{\prime}}{\mathcal{S}_{q}^{\kappa, k} \psi(z)}\right)-\frac{1}{z}=\frac{h(ð(z))-1}{z} .
$$

By integrating both sides, we obtain

$$
\log \mathcal{S}_{q}^{\kappa, k} v(z)-\log z=\int_{0}^{z} \frac{h(ð(w))-1}{w} d w .
$$

Thus, we have

$$
\log \frac{\mathcal{S}_{q}^{\kappa, k} v(z)}{z}=\int_{0}^{z} \frac{h(ð(\xi))-1}{w} d w
$$

By utilizing the meaning of subordination, we conclude that

$$
\mathcal{S}_{q}^{\kappa, k} v(z) \prec z \exp \left(\int_{0}^{z} \frac{h(ð(w))-1}{w} d w\right)
$$

Besides, we find that the function $h(z)$ maps the disk $0<|z|<\chi<1$ onto a domain which is convex and symmetric with respect to the real axis, which means

$$
h(-\chi|z|) \leq \Re(h(ð(\chi z))) \leq h(\chi|z|), \quad \chi \in(0,1),
$$

then we obtain the next relations:

$$
h(-\chi) \leq h(-\chi|z|), \quad h(\chi|z|) \leq h(\chi),
$$

and

$$
\int_{0}^{1} \frac{h(ð(-\chi|z|))-1}{\chi} d \chi \leq \Re\left(\int_{0}^{1} \frac{h(ð(\chi))-1}{\chi} d \chi\right) \leq \int_{0}^{1} \frac{h(ð(\chi|z|))-1}{\chi} d \chi
$$

By employing Eq. (5), we deduce that

$$
\int_{0}^{1} \frac{h(ð(-\chi|z|))-1}{\chi} d \chi \leq \log \left|\frac{\mathcal{S}_{q}^{\kappa, k} v(z)}{z}\right| \leq \int_{0}^{1} \frac{h(ð(\chi|z|))-1}{\chi} d \chi
$$

which leads to

$$
\exp \left(\int_{0}^{1} \frac{h(ð(-\chi|z|))-1}{\chi} d \chi\right) \leq\left|\frac{\mathcal{S}_{q}^{\kappa, k} v(z)}{z}\right| \leq \exp \left(\int_{0}^{1} \frac{h(ð(\chi|z|))-1}{\chi} d \chi\right) .
$$

Hence, we have

$$
\exp \left(\int_{0}^{1} \frac{h(ð(-\chi))-1}{\eta}\right) d \chi \leq\left|\frac{\mathcal{S}_{q}^{\kappa, k} v(z)}{z}\right| \leq \exp \left(\int_{0}^{1} \frac{h(ð(\chi))-1}{\chi}\right) d \chi .
$$


Corollary 3.1 ([8]) Let $q \longrightarrow 1$ in Theorem 3.2. Then

$$
\mathcal{S}_{1}^{\kappa, k} v(z) \prec z \exp \left(\int_{0}^{z} \frac{h(ð(w))-1}{w} d w\right) .
$$

Note that all the special cases of the class $S_{q}^{*}(\kappa, k, h)$ can be considered as consequences of Theorem 3.2.

Theorem 3.3 If $v \in \mathbb{J}_{q}^{\kappa, b}(A, B, k)$ then the odd function

$$
\mathfrak{B}(z)=\frac{1}{2}[v(z)-v(-z)], \quad z \in \mathbb{U}
$$

attains the subordination inequalities

$$
1+\frac{1}{b}\left(\frac{\mathcal{S}_{q}^{\kappa, k+1} \mathfrak{B}(z)}{\mathcal{S}_{q}^{\kappa, k} \mathfrak{B}(z)}-1\right) \prec \frac{1+A z}{1+B z}
$$

and

$$
\begin{aligned}
& \Re\left(\frac{z \mathfrak{B}(z)^{\prime}}{\mathfrak{B}(z)}\right) \geq \frac{1-\varrho^{2}}{1+\varrho^{2}}, \quad|z|=\varrho<1, \\
& \quad(z \in \mathbb{U},-1 \leq B<A \leq 1, k=1,2, \ldots, b \in \mathbb{C} \backslash\{0\}, \kappa \in \mathbb{R}) .
\end{aligned}
$$

Proof Let $v \in \mathbb{J}_{q}^{\kappa, b}(A, B, k)$. Then there exists a function $P \in \mathbb{J}(A, B)$ with the layout

$$
b(P(z)-1)=\left(\frac{2 \mathcal{S}_{q}^{\kappa, k+1} v(z)}{\mathcal{S}_{q}^{\kappa, k} v(z)-\mathcal{S}_{q}^{\kappa, k} v(-z)}\right)
$$

and

$$
b(P(-z)-1)=\left(\frac{-2 \mathcal{S}_{q}^{\kappa, k+1} v(-z)}{\mathcal{S}_{q}^{\kappa, k} v(z)-\mathcal{S}_{q}^{\kappa, k} v(-z)}\right) .
$$

This yields

$$
1+\frac{1}{b}\left(\frac{\mathcal{S}_{q}^{\kappa, k+1} \mathfrak{B}(z)}{\mathcal{S}_{q}^{\kappa, k} \mathfrak{B}(z)}-1\right)=\frac{P(z)+P(-z)}{2} .
$$

In addition, since $P$ fulfills the inequality

$$
P(z) \prec \frac{1+A z}{1+B z}
$$

where $\frac{1+A z}{1+B z}$ is univalent, by the idea of subordination, we obtain

$$
1+\frac{1}{b}\left(\frac{\mathcal{S}_{q}^{\kappa, k+1} \mathfrak{B}(z)}{\mathcal{S}_{q}^{\kappa, k} \mathfrak{B}(z)}-1\right) \prec \frac{1+A z}{1+B z} .
$$


Also, the odd function $\mathfrak{B}(z)$ is starlike in $\mathbb{U}$, which produces the subordination inequality

$$
\frac{z \mathfrak{B}(z)^{\prime}}{\mathfrak{B}(z)} \prec \frac{1-z^{2}}{1+z^{2}}
$$

that is, there exists a Schwarz function $\gamma \in \mathbb{U},|\gamma(z)| \leq|z|<1, \gamma(0)=0$ with the property

$$
\Xi(z):=\frac{z \mathfrak{B}(z)^{\prime}}{\mathfrak{B}(z)} \prec \frac{1-\gamma(z)^{2}}{1+\gamma(z)^{2}},
$$

which leads to

$$
\gamma^{2}(\zeta)=\frac{1-\Xi(\zeta)}{1+\Xi(\zeta)}, \quad \zeta \in \mathbb{U}, \zeta,|\zeta|=r<1
$$

A computation implies that

$$
\left|\frac{1-\Xi(\zeta)}{1+\Xi(\zeta)}\right|=|\gamma(\zeta)|^{2} \leq|\zeta|^{2} .
$$

Therefore, we get the following inequality:

$$
\left|\Xi(\zeta)-\frac{1+|\zeta|^{4}}{1-|\zeta|^{4}}\right|^{2} \leq \frac{4|\zeta|^{4}}{\left(1-|\zeta|^{4}\right)^{2}}
$$

or

$$
\left|\Xi(z)-\frac{1+|\zeta|^{4}}{1-|\zeta|^{4}}\right| \leq \frac{\left.2 \zeta\right|^{2}}{\left(1-|\zeta|^{4}\right)}
$$

Consequently, we obtain the result

$$
\Re(\Xi(z)) \geq \frac{1-\varrho^{2}}{1+\varrho^{2}}, \quad|\zeta|=\varrho<1 .
$$

The following consequences of Theorem 3.3 can be found in $[26,27]$ and [8], respectively.

Corollary 3.2 Let $\lambda=1$ in Theorem 3.3. Then

$$
1+\frac{1}{b}\left(\frac{\mathcal{S}_{q}^{0, k+1} \mathfrak{B}(z)}{\mathcal{S}_{q}^{0, k} \mathfrak{B}(z)}-1\right) \prec \frac{1+A z}{1+B z} .
$$

Corollary 3.3 Let $\kappa=0, k=1$ and $q \longrightarrow 1$ in Theorem 3.3. Then

$$
1+\frac{1}{b}\left(\frac{\mathcal{S}_{q}^{0,2} \mathfrak{B}(z)}{\mathcal{S}_{q}^{0,1} \mathfrak{B}(z)}-1\right) \prec \frac{1+A z}{1+B z} .
$$

Corollary 3.4 Let $q \longrightarrow 1$ in Theorem 3.3. Then

$$
1+\frac{1}{b}\left(\frac{\mathcal{S}_{q}^{\kappa, k+1} \mathfrak{B}(z)}{\mathcal{S}_{q}^{\kappa, k} \mathfrak{B}(z)}-1\right) \prec \frac{1+A z}{1+B z} .
$$




\section{Applications}

We produce a presentation of our results established by the solution of the complex BriotBouquet (BB) differential equation [11]. The class of complex Briot-Bouquet differential equations is a link of differential equations whose consequences are visible in the complex plane. Accruing integrals shade special paths to follow, which have singularities and branch points of the equation we must study. Existence and uniqueness theorems contain the efficacy of upper and lower (subordination and superordination relations) (see [29-32]). The study of the rational first ODEs in the complex domain indicates new transcendental special functions as follows:

$$
\beta v(z)+(1-\beta) \frac{z(v(z))^{\prime}}{v(z)}=h(z), \quad h(0)=v(0), \quad \beta \in[0,1] .
$$

Many applications of these equations in geometric function theory have newly been researched in [11]. Our goal is to propagate this class of equations by applying the suggested operator and establishing its solutions using the subordination relations. The q-SDD in (2) propagates the complex Briot-Bouquet differential equation as follows:

$$
\beta v(z)+(1-\beta)\left(\frac{z\left(\mathcal{S}_{q}^{\kappa, k} v(z)\right)^{\prime}}{\mathcal{S}_{q}^{\kappa, k} v(z)}\right)=h(z), \quad h(0)=v(0), \quad z \in \mathbb{U} .
$$

The subordination conditions and distortion bounds for a class of complex conformable fractional derivative are given in the next theorem. A trivial solution of (6) is given when $\beta=1$. Therefore, our study concerns the case with $v \in \Lambda$ and $\beta=0$.

Theorem 4.1 Consider Eq. (6) with $\beta=0$ and $\psi \in \Lambda$ with non-negative coefficients. If $h(z), z \in \mathbb{U}$ is univalent convex in $\mathbb{U}$ then there exists a solution satisfying the subordination (major solution)

$$
\mathcal{S}_{q}^{\kappa, k} v(z) \prec z \exp \left(\int_{0}^{z} \frac{h(ð(w))-1}{w} d w\right),
$$

where $\partial(z)$ is analytic in $\mathbb{U}$, with $\partial(0)=0$ and $|\partial(z)|<1$.

Proof Collect all the assumptions of Eq. (6), and $v(z) \in \Lambda$. Then we get the following conclusion:

$$
\begin{aligned}
& \Re\left(\frac{z\left(\mathcal{S}_{q}^{\kappa, k} v(z)\right)^{\prime}}{\mathcal{S}_{q}^{\kappa, k} v(z)}\right)>0 \\
& \Leftrightarrow \quad \Re\left(\frac{z+\sum_{n=2}^{\infty} n\left([n, q]+\frac{\kappa}{2}\left(1+(-1)^{n+1}\right)\right)^{k} \vartheta_{n} z^{n}}{z+\sum_{n=2}^{\infty}\left([n, q]+\frac{\kappa}{2}\left(1+(-1)^{n+1}\right)\right)^{k} \vartheta_{n} z^{n}}\right)>0 \\
& \Leftrightarrow \quad \Re\left(\frac{1+\sum_{n=2}^{\infty} n\left([n, q]+\frac{\kappa}{2}\left(1+(-1)^{n+1}\right)\right)^{k} \vartheta_{n} z^{n-1}}{1+\sum_{n=2}^{\infty}\left([n, q]+\frac{\kappa}{2}\left(1+(-1)^{n+1}\right)\right)^{k} \vartheta_{n} z^{n-1}}\right)>0 \\
& \Leftrightarrow \quad\left(\frac{1+\sum_{n=2}^{\infty} n\left([n, q]+\frac{\kappa}{2}\left(1+(-1)^{n+1}\right)\right)^{k} \vartheta_{n}}{1+\sum_{n=2}^{\infty}\left([n, q]+\frac{\kappa}{2}\left(1+(-1)^{n+1}\right)\right)^{k} \vartheta_{n}}\right)>0, \quad z \rightarrow 1^{+} \\
& \Leftrightarrow \quad\left(1+\sum_{n=2}^{\infty} n\left([n, q]+\frac{\kappa}{2}\left(1+(-1)^{n+1}\right)\right)^{k} \vartheta_{n}\right)>0 .
\end{aligned}
$$


Moreover, by the definition of $\mathcal{S}_{q}^{\kappa, k} v(z)$, we indicate that $\left(\mathcal{S}_{q}^{\kappa, k} v\right)(0)=0$. Consequently,

$$
\frac{z\left(\mathcal{S}_{q}^{\kappa, k} v(z)\right)^{\prime}}{\mathcal{S}_{q}^{\kappa, k} v(z)} \in \mathcal{P} \quad \Rightarrow \quad v(z) \in S_{q}^{*}(\kappa, k, h)
$$

Hence, in view of Theorem 3.2, we have the desired result (7).

\section{Conclusion}

By our method, we have revealed new classes of univalent functions, which assign a q-SDD operator in the open unit disk. We obtained appropriate essential conditions of these subclasses. Applications involved the BB equation and investigated its solution in the open unit disk. For further study, we encourage researchers to introduce some certain new classes related to other kinds of analytic functions such as harmonic, symmetric, p-valent and meromorphic functions with respect to symmetric points associated by (2).

Acknowledgements

The authors would like to express their thanks to the reviewers to provide us with deep comments.

\section{Funding}

The work here is partially supported by the Universiti Kebangsaan Malaysia grant: GUP (Geran Universiti Penyelidikan)-2019-032.

Competing interests

The authors declare no conflict of interest.

\section{Authors' contributions}

Conceptualization was by RWI and MD; the methodology by RWl; the validationby RWI and MD; the formal analysis by RWI and MD; investigation by RWI and MD; writing and original draft preparation by RWl; writing review and editing by MD. All authors read and approved the final manuscript.

\section{Author details}

${ }^{1}$ Informetrics Research Group, Ton Duc Thang University, Ho Chi Minh City, Vietnam. ${ }^{2}$ Faculty of Mathematics \& Statistics, Ton Duc Thang University, Ho Chi Minh City, Vietnam. ${ }^{3}$ Centre for Modelling and Data Science, Faculty of Science and Technology, Universiti Kebangsaan Malaysia, Bangi, Malaysia.

\section{Publisher's Note}

Springer Nature remains neutral with regard to jurisdictional claims in published maps and institutional affiliations.

Received: 16 August 2019 Accepted: 3 December 2019 Published online: 12 December 2019

\section{References}

1. Carmichael, R.D.: The general theory of linear q-difference equations. Am. J. Math. 34, 147-168 (1912)

2. Jackson, F.H.: On q-definite integrals. Quart. J. Pure Appl. Math. 41, 193-203 (1910)

3. Mason, T.E.: On properties of the solution of linear q-difference equations with entire function coefficients. Am. J. Math. 37, 439-444 (1915)

4. Trjitzinsky, W.J.: Analytic theory of linear q-difference equations. Acta Math. 61, 1-38 (1933)

5. Ismail, M.E.H., Merkes, E., Styer, D.: A generalization of starlike functions. Complex Var. Theory Appl. 14, 77-84 (1990)

6. Sàlàgean, G.S.: Subclasses of univalent functions. In: Complex Analysis-Fifth Romanian-Finnish Seminar, Part 1, Bucharest, 1981. Lecture Notes in Math., vol. 1013, pp. 362-372. Springer, Berlin (1983)

7. Al-Oboudi, F.M.: On univalent functions defined by a generalized Sàlàgean operator. Int. J. Math. Math. Sci. 2004(27), 1429-1436 (2004)

8. Ibrahim, R.W., Darus, M.: Univalent functions formulated by the Salagean-difference operator. Int. J. Anal. Appl. 17(4), 652-658 (2019)

9. Naeem, M., et al.: A new subclass of analytic functions defined by using Salagean q-differential operator. Mathematics 7(5), 458 (2019)

10. Duren, P.: Univalent Functions. Grundlehren der Mathematischen Wissenschaften, vol. 259. Springer, New York (1983)

11. Miller, S.S., Mocanu, P.T.: Differential Subordinations: Theory and Applications. CRC Press, Boca Raton (2000)

12. Govindaraj, M., Sivasubramanian, S.: On a class of analytic functions related to conic domains involving q-calculus. Anal. Math. 43, 475-487 (2017)

13. Dunkl, C.F.: Differential-difference operators associated to reflection groups". Trans. Am. Math. Soc. 311(1), 167-183 (1989) 
14. Jakubowski, Z.J., Kaminski, J.: On some properties of Mocanu-Janowski functions. Rev. Roum. Math. Pures Appl. 23, 1523-1532 (1978)

15. Obradović, M., Owa, S.: On certain properties for some classes of starlike functions. J. Math. Anal. Appl. 145(2), 357-364 (1990)

16. Ma, W.C., Minda, D.: A unified treatment of some special classes of univalent functions. In: Proceedings of the Conference on Complex Analysis, Tianjin, China, pp. 19-23 (1992)

17. Goel, P., Kumar, S.S.: Certain Class of Starlike Functions Associated with Modified Sigmoid Function. Bull. Malays. Math Sci. Soc., 1-35 (2019)

18. Dziok, J., Raina, R.K., Sokół, J.: Certain results for a class of convex functions related to a shell-like curve connected with Fibonacci numbers. Comput. Math. Appl. 61, 2605-2613 (2011)

19. Dziok, J., Raina, R.K., Sokół, J.: On a class of starlike functions related to a shell-like curve connected with Fibonacci numbers. Math. Comput. Model. 57, 1203-1211 (2013)

20. Kargar, R., Ebadian, A., Sokol, J.: On subordination of some analytic functions. Sib. Math. J. 57, 599-605 (2016)

21. Kargar, R., Ebadian, A., Sokół, J.: On booth lemniscate and starlike functions. Anal. Math. Phys. 9(1), 143-154 (2019)

22. Sokół, J.: On some subclass of strongly starlike functions. Demonstr. Math. 21, 81-86 (1998)

23. Cho, N.E., Kumar, V., Kumar, S.S., Ravichandran, V.: Radius problems for starlike functions associated with the sine function. Bull. Iran. Math. Soc. 45, 213-232 (2019)

24. Tang, $\mathrm{H}$., et al.: Majorization results for subclasses of starlike functions based on the sine and cosine functions. Bull. Iran. Math. Soc., 1-8 (2019)

25. Sivasubramanian, S., et al.: Differential subordination for analytic functions associated with left-like domains. J. Comput. Anal. Appl. 26(1) (2019)

26. Arif, M., et al.: A new class of analytic functions associated with Sălăgean operator. J. Funct. Spaces 2019, Article ID 6157394 (2019). https://doi.org/10.1155/2019/6157394

27. Sakaguchi, K.: On a certain univalent mapping. J. Math. Soc. Jpn. 11, $72-75$ (1959)

28. Das, R.N., Singh, P.: On sub classes of Schlicht mapping. Indian J. Pure Appl. Math. 8, 864-872 (1977)

29. Ibrahim, R.W., Darus, M.: Subordination and superordination for univalent solutions for fractional differential equations. J. Math. Anal. Appl. 345(2), 871-879 (2008)

30. Ibrahim, R.W.: On holomorphic solutions for nonlinear singular fractional differential equations. Comput. Math. Appl. 62(3), 1084-1090 (2011)

31. Ibrahim, R.W.: Existence and uniqueness of holomorphic solutions for fractional Cauchy problem. J. Math. Anal. Appl. 380(1), 232-240 (2011)

32. Ibrahim, R.W.: Fractional complex transforms for fractional differential equations. Adv. Differ. Equ. 2012(1), 192 (2012)

\section{Submit your manuscript to a SpringerOpen ${ }^{\circ}$ journal and benefit from:}

- Convenient online submission

- Rigorous peer review

- Open access: articles freely available online

- High visibility within the field

Retaining the copyright to your article

Submit your next manuscript at $\gg$ springeropen.com 